\title{
Circoncision féminine et masculine en pays kikuyu
}

Rite d'institution, division sociale et droits de l'Homme

Male and Female Circumcision among the Kikuyu: A Rite of Institution, Social Division and Human Rights

\section{Yvan Droz}

\section{OpenEdition}

\section{Journals}

Édition électronique

URL : http://journals.openedition.org/etudesafricaines/172

DOI : $10.4000 /$ etudesafricaines. 172

ISSN : $1777-5353$

Éditeur

Éditions de l'EHESS

\section{Édition imprimée}

Date de publication : 1 janvier 2000

Pagination : $215-240$

ISBN : 978-2-7132-1356-4

ISSN : 0008-0055

Référence électronique

Yvan Droz, "Circoncision féminine et masculine en pays kikuyu », Cahiers d'études africaines [En ligne], 158 | 2000, mis en ligne le 20 novembre 2013, consulté le 05 février 2021. URL : http:// journals.openedition.org/etudesafricaines/172 ; DOI : https://doi.org/10.4000/etudesafricaines. 172 


\title{
Yvan Droz
}

\section{Circoncision féminine et masculine en pays kikuyu :}

\author{
rite d'institution, division sociale et droits de l'Homme*
}

Il s'agit de présenter les aspects anthropologiques du rituel de la circoncision chez les Kikuyu et de proposer, d'une part un bref rappel de la situation politique du Kenya au moment où certains missionnaires se sont attaqués à la reproduction sociale kikuyu en général et à la circoncision féminine et masculine en particulier. D'autre part, nous nous interrogerons sur les effets anthropologiques de l'interdiction de la circoncision féminine au Kenya en comparant sa pratique actuelle avec celle de la circoncision masculine. Pour cela, l'ethnographie des classes d'âge kikuyu et de leur fonction dans l'organisation sociale s'avère être un préalable indispensable. En effet, comprendre une pratique sociale particulière - la circoncision - exige de connaître les relations qu'elle entretient avec l'ensemble de la reproduction sociale. Nous montrerons que si la circoncision masculine reste légale, elle ne crée plus de classe d'âge, mais institue toujours le jeune homme comme adulte Kikuyu. La circoncision féminine, bien qu'interdite, est toujours pratiquée par de nombreux Kikuyus. Pourtant, le secret où elle se trouve cantonnée crée un doute sur la qualité de la femme kikuyu et divise profondément les communautés rurales. En d'autres termes, la circoncision tant masculine que féminine ne contrecarre plus les aspects fissionnels de la parenté au sein de l'organisation sociale, et son interdiction contribue à déprécier le statut de la femme kikuyu qui n'est plus instituée socialement en tant que femme adulte. Quelques remarques sur le changement de perspective qu'implique cette analyse souligneront les présupposés individualistes et la conception du citoyen à l'origine des tentatives d'interdiction de

* Cet article s'appuie sur la recherche Family Networks, Security of Subsistence and Social Differentiation in the Northwestern Footzones of Mount Kenya (NSD), financée par le Fonds national suisse de la recherche scientifique (FNSRS ; crédits 12-33732.92 et 12-042005.94). Nous remercions Nicolas Guillaume-Gentil, Alessandro Monsutti, Doris Nyfeler, Véronique Pache et Gilbert Rist qui, par leurs remarques et critiques, nous ont permis d'améliorer ce texte. Pourtant, si nous leur sommes redevable pour la clarté de nos propos, nous restons le seul responsable des maladresses et des insuffisances de cet article. 
la circoncision féminine au nom des droits de l'Homme. En d'autres termes, cette focalisation sur les aspects individuels de la clitoridectomie interdit de considérer les principes anthropologiques de la circoncision féminine en raison de l'outrance affective qu'elle suscite.

Précisons d'emblée qu'un seul et même terme kikuyu (irua) s'appliquait au rite de passage, synonyme de l'entrée dans l'âge adulte et symbolisé par la circoncision des candidats des deux sexes. C'est pourquoi nous utiliserons le terme de «circoncision » pour la clitoridectomie et pour la circoncision masculine, suivant en cela l'usage kenyan du terme anglais circumcision. Par ailleurs, nous avons préféré le terme de « circoncision féminine » à celui d'excision pour souligner l'interdépendance essentielle qui existe entre les circoncisions féminines et masculines, au contraire de ce qu'affirme trop rapidement Nicole Sindzingre ${ }^{1}$. Ce rituel, paradigme du rite de passage, inscrivait le nouveau circoncis (ou la nouvelle circoncise) dans le monde des individus responsables d'eux-mêmes - habilités à respecter les convenances et à reproduire la société, tant biologiquement que socialement et constituait la classe d'âge ${ }^{2}$.

Afin d'éviter tout malentendu quant à l'objet de notre propos, une précision s'impose. Chez les Kikuyus, l'opération physique est limitée à la clitoridectomie mineure qui consiste à exciser le capuchon clitoridien. Elle s'accompagne parfois de l'ablation partielle du clitoris et de celle des petites lèvres, mais il n'a jamais été question d'infibulation (Browne 1991). Louis Leakey (1977 : 622) décrit l'opération en ces termes : «With her [the female operator] left hand she parted the labia majora and took hold of the clitoris between the thumb and first finger of her left hand. Pulling it forward as much as she could, she cut off a tiny portion of the skin, using a special sharp razor to do so. [...] Sometimes the operator also excised a little piece of the labia majora on either side of the clitoris. » On peut la comparer à celle des jeunes hommes : «[The operator] then cut a slit across the hinder portion of the prepuce on the upper surface and at right angles to the penis. Having made this slit, he brought the glans penis up through the slit so that it was exposed. The foreskin was not cut off but was left below the glans penis, where it remained for the rest of his life » (ibid. : 621). Soulignons que la peau du pénis cicatrisée formait une boule censée augmenter

1. Les arguments que cette auteure présente sont infirmés par l'exemple des Kikuyus. Il est donc capital de considérer, comme elle l'affirme en fin d'article (SINDZINGRE 1977: 74), chaque cas particulier de circoncision - au sens large - avant d'élaborer une théorie générale. Notons que Nicole Sindzingre présente une position plus nuancée dans un article ultérieur. En accord avec elle (id. 1979 : 178), il nous paraît tout aussi essentiel de ne pas regrouper au sein d'un même ensemble la clitoridectomie mineure, la clitoridectomie majeure et l'infibulation, car chacune de ces pratiques relève d'une conceptualisation particulière.

2. Le lecteur intéressé trouvera une description minutieuse et «traditionnaliste »du rituel chez L. S. B. LEAKEY (1977 : 587-686) qu'il pourra comparer à celle de R. J. Bunche (1941) et de F. Bugeau (1911) et aux récits rapportés par J. DAVISON (1989). 
la jouissance de la femme lors des relations sexuelles; de même, l'ablation partielle du clitoris ou de son capuchon était censée augmenter la jouissance des femmes circoncises. L'hypothèse qui considère la circoncision féminine comme un moyen de limiter la jouissance sexuelle de la femme ne s'applique donc pas au cas des Kikuyus.

En outre, cette manière de circoncire les jeunes hommes et les jeunes femmes se distinguait de celle des autres groupes locaux. Il s'agissait donc également d'un marqueur identitaire rattaché au registre ethnique, puisque le type de circoncision en pays kikuyu, s'il pouvait varier, correspondait à une structure commune qui se distinguait de celle en vigueur chez les Maasaï ou chez les Kamba par exemple. Ceci souligne l'importance d'une connaissance ethnographique précise pour comprendre le sens et les conséquences d'une pratique sociale, par définition contextualisée. Les différents types de clitoridectomie présents en Afrique occidentale ou en Afrique du Nord par exemple ne correspondent pas à la problématique de la circoncision kikuyu. Ainsi, nos propos ne s'appliquent pas à la clitoridectomie en tant que telle, mais bien à celle qui est pratiquée parmi les Kikuyus.

\section{La controverse sur la clitoridectomie}

Un rappel lapidaire du déroulement de la controverse sur la clitoridectomie dans les années vingt et les années trente ${ }^{3}$ est un préalable indispensable à cette présentation. En 1929, à la veille de l'introduction dans la colonie du Kenya d'un nouveau code pénal destiné à remplacer le code indien, la Church of Scotland Mission (CSM), sous la direction du Dr. Arthur, entame une croisade pour que l'interdiction de la circoncision féminine soit inscrite dans le nouveau code. A la suite du mécontentement que suscitent les velléités «législatrices » de la CSM, rapidement rejointe par deux autres Églises missionnaires (Africa Inland Mission et Gospel Missionary Society), la Kikuyu Central Association s'oppose ostensiblement aux prétentions des missionnaires de régir les «traditions » kikuyus et trouve là un moyen d'étendre son influence parmi la population kikuyu.

Pour les missionnaires fondamentalistes, la clitoridectomie faisait partie d'un ensemble de pratiques païennes qu'il s'agissait d'éradiquer (Browne 1991 : 256-257). Ainsi en était-il des danses qui suivaient le rite de passage où les propos licencieux, voire irrévérencieux, étaient monnaie courante. Les pratiques thérapeutiques «traditionnelles » étaient condamnées, à l'instar des sacrifices incompatibles avec le christianisme. Pour les Kikuyus, il s'agissait d'une attaque directe contre la «kikuyuité » - l'identité ethnique — qui

3. Une littérature abondante existe concernant la controverse sur la clitoridectomie. Nous renvoyons le lecteur aux textes de B. M. Ahlberg (1991), J. Davison (1989), T. Kanogo (1993), J. Lonsdale (1992: 388-395), J. Murray (1974), V. Neckebrouck (1978: 208-245), D. P. Sandgren (1989), R. W. Strayer (1978). 
mettait en péril la reproduction sociale des groupes locaux. Ils ont compris cette tentative de supprimer le rituel comme un nouveau moyen inventé par les colons pour s'approprier les terres kikuyus. En effet, si les jeunes gens ne devenaient pas des adultes kikuyus, grâce à la circoncision, les sacrifices destinés aux ancêtres, identifiés à la propriété foncière, ne pourraient plus avoir lieu. En l'absence de la protection des «esprits» de la terre, le domaine familial n'existait plus en tant que tel et pouvait être vendu.

L'exemple d'une rumeur permet de saisir le climat qui enveloppait la controverse sur la clitoridectomie dans les années trente. «On» imputait aux missionnaires et aux colons le projet d'annihiler les Kikuyus — pour les déposséder de leur terre - en supprimant la clitoridectomie, ce qui empêcherait les femmes de mettre au monde des enfants «normaux », i.e. de «vrais » Kikuyus. Ce projet devait déboucher sur l'interdiction de la circoncision masculine et mettre ainsi un terme à l'existence des Kikuyus en tant que groupe ethnique, puisque seul un circoncis peut devenir un adulte (Neckebrouck 1978: 382-390). L'assimilation de la terre à la famille est au cœur de l'ethos kikuyu, comme la circoncision est au cœur de l'identité kikuyu (Kershaw 1997: 66-68; Droz 1999). Pour les Kikuyus, abolir la circoncision féminine était donc considéré comme une première étape vers la disparition des Kikuyus en tant que groupe ethnique, vers leur dissolution dans la masse informe des people of different cultures (Davison 1989 : 201).

Des pressions officielles et officieuses sont exercées de part et d'autres et conduisent à une importante agitation politique. Télégrammes, pétitions, auditions, mémorandums destinés tant aux autorités religieuses qu'au Colonial Office, se succèdent. Après quelques atermoiements et tergiversations, la situation politique s'apaise sans qu'une modification légale ne soit entrée en vigueur. Néanmoins, les trois Églises au cœur de la croisade contre la clitoridectomie édictent des règlements internes prohibant cette pratique, et, par contrecoup donne naissance aux Églises et aux écoles indépendantes ${ }^{4}$. En effet, nombre de fidèles, partisans de la circoncision féminine, quittent les Églises missionnaires et fondent de nouveaux mouvements religieux qui, alliés aux associations d'écoles indépendantes, seront promis à un fécond avenir.

Bref, les résultats de cette croisade peuvent se résumer en quelques points. En premier lieu, une polarisation des pratiques sociales conduit, d'une part les adhérents des Églises anti-clitoridectomie à condamner sans appel cette pratique, et, d'autre part les autres Kikuyus à considérer la circoncision féminine comme un élément essentiel à l'identité ethnique ; en deuxième lieu, les nouvelles Églises indépendantes saignent les Églises missionnaires de leurs fidèles et donnent naissance à un système scolaire autochtone ; en dernier lieu, le développement rapide de la popularité de la

4. Sur le mouvement des écoles indépendantes, voir T. KANOGO (1987), T. NATSOUlas (1988), V. Neckebrouck (1978, 1983), D. P. Sandgren (1989), R. Strayer (1978), R. L. Tignor (1976). 
Kikuyu Central Association au Kenya conduit à sa reconnaissance officieuse par le Colonial Office à Londres. Pour sa part, l'administration coloniale kenyane considère qu'il est « urgent de ne rien faire » et ne mentionne pas la circoncision féminine dans le nouveau code pénal.

La résistance à l'abolition de la circoncision féminine a traversé le siècle. Plusieurs tentatives pour interdire cette pratique ont alimenté le «nationalisme kikuyu » lors des situations de crises telles que la Seconde Guerre mondiale ou la Guerre civile des Mau Mau. En effet, même les pouvoirs importants reçus par les autorités administratives, associées à la volonté d'un groupe, certes minoritaire, de loyalistes, n'ont pas suffi pour imposer une modification légale dans un district de la province centrale, encore moins dans l'ensemble du Kenya (Murray 1974: 318-327). La croisade anticlitoridectomie a constitué un échec, voire une déroute pour les Églises qui l'ont animée. Pourtant, quelques Kikuyus ont effectivement abandonné cette pratique, souvent au prix de l'opprobre de leurs voisins.

Après l'Indépendance, la situation s'est peu modifiée, bien qu'un nombre croissant de jeunes femmes aient cessé de se faire circoncire. Dès les années soixante-dix, le quart des jeunes filles kikuyus ne seront plus circoncises, alors que la clitoridectomie s'adaptera sans difficulté à l'urbanisation. En 1982, sous la pression internationale, le président Daniel Arap Moi interdira la clitoridectomie (Murray 1974 : 352 ; Browne 1991 ; Davison $1989: 202)^{5}$.

\section{Imaginons les différentes dimensions de l'organisation sociale}

Pour comprendre les raisons de l'opposition farouche à l'interdiction de la clitoridectomie en pays kikuyu et les conséquences de son abandon progressif, il est nécessaire de décrire rapidement l'organisation sociale dans laquelle cette pratique jouait un rôle important. Ce long détour est la condition d'un regard anthropologique sur la clitoridectomie kikuyu. L'organisation sociale kikuyu se fondait sur trois principes généraux: le régime générationnel, le régime des classes d'âge et le régime de la parenté. A ces trois régimes structurants qui constituaient autant de registres identitaires pour les agents sociaux, il faut encore ajouter une prétention à l'accomplissement personnel exprimée par l'ethos du mûramati (Droz 1999, 2000 ; Peatrik 1994). Cette constellation particulière de principes organisationnels est propre aux Kikuyus précoloniaux et les distingue de leurs voisins proches (Peatrik 1994: 30).

A la verticale, nous trouvons le régime de parenté avec le «clan »mûhîrîga, qui regroupe de nombreux «lignages » mbarî, composés de familles étendues. Celles-ci rassemblent des familles patrimoniales, simple unité

5. Cette interdiction repose sur un discours du président et non pas sur un texte législatif (GunNING 1992 : 227-228). 
domestique nyûmba, ou groupe d'unités domestiques. Cette verticale de la parenté est doublée par une verticale territoriale qui subdivise le schéma en régions (Gaki, Metumi...), elles-mêmes divisées en interfluve rûgongo ou itûura. Les interfluves se distribuent en «feux» mwaki composés de plusieurs unités domestiques réunies par la transmission du feu de cuisine : il s'agit des relations de voisinage. Celles-ci étaient, certes, ancrées dans la parenté, mais aussi dans le régime générationnel (les cérémonies rassemblaient des régions entières) et dans le régime des classes d'âge (les circoncisions s'accomplissaient par interfluves). Néanmoins, ces relations de voisinage étaient déterminées principalement par la proximité territoriale, par le relief (crêtes ou interfluves) et les cours d'eau.

La diagonale représente le régime générationnel où les générations Mwangi alternent avec celles des Maina. Ce régime traverse tant les relations de parenté que les classes d'âge. Chaque génération regroupe des individus d'âge fort différent, ce régime traverse tant les classes d'âge que la verticale des âges biologiques ou des relations de parenté ou de voisinage.

L'horizontale incarne le régime des classes d'âge dont les régiments sont composés de neuf classes d'âge et d'une période de marge, sans promotion. Ensemble, l'horizontale et la diagonale présentent donc la complexité du riika sous ses trois aspects différents : promotion ou classe d'âge, régiment et génération ${ }^{6}$, sans doute trop polarisés par des traductions distinctes. En effet :

«Un seul terme (riika, mariika) désigne en kikuyu les trois sortes de classes auxquelles tout homme appartient simultanément: a) la classe des individus initiés la même année que l'on appellera "promotion" pour plus de commodité ; b) l'ensemble des promotions recrutées durant un certain laps de temps que nous désignerons par "classe régimentaire", appellation calquée sur celle de l'anglais regiment qui nous semble bien refléter la fonction, pour une fois effectivement guerrière de ces classes ; c) les classes de génération » (Peatrik 1994: 9).

En outre, deux aspects du riika sont attachés à des représentations distinctes du territoire, ce qui inscrit le riika dans la géographie, à l'instar de la parenté (Neckebrouck 1983 : 353-354 ; Kershaw 1997 : 18). Les générations règnent sur une des deux notions du territoire (le bûrûri), alors que les régiments contrôlent une autre conception du territoire (le thî̀), sans doute plus pragmatique puisqu'il s'agissait d'y faire régner l'ordre public (Peatrik 1994). Deux temps sont également à distinguer : le temps linéaire - qui est également celui du phylum familial - qu'expriment les régiments (chacun d'eux recevait un nom singulier); le temps semi-cyclique des générations qui se répétaient à l'identique. Pourtant, chaque génération recevait

6. En ce qui concerne les riika composés de plusieurs promotions qui constituent les régiments, voir H. E. LAMBERT (1956: 8-31) ; pour les classes générationnelles (mwangi et maina) auxquelles la littérature se réfère souvent par le terme d'ituîka, voir A.-M. PeatriK (1994); J. Lonsdale $(1992,1995)$ et G. Kershaw (1997). 
un nom propre une fois que tous ses membres avaient disparu. Ce temps répétitif s'exprime dans la dation des noms individuels, reproduction incessante des mêmes noms au sein du «lignage». Une représentation peutêtre plus adéquate de ces deux temps pourrait prendre la forme d'une ligne hélicoïdale : temps générationnel et temps du phylum familial ne formant qu'une flèche qui revient sur elle-même (les générations) tout en s'élevant (les classes d'âge et les régiments).

Ainsi, les trois régimes que nous avons évoqués se distribuent dans trois dimensions : verticale pour la parenté, horizontale pour les classes d'âge, et diagonale pour les générations. L'individu se trouve à la croisée de ces trois dimensions, et son être social est défini par elles. Cette complexité où il est facile, voire légitime, de se perdre souligne bien l'éventail de registres identitaires dont disposaient les individus dans cette organisation sociale complexe (Droz 1998).

Pour compléter ce système complexe, il faudrait ajouter la hiérarchie des différents conseils (kiama) avec, d'un côté ceux des femmes, et de l'autre ceux des hommes ${ }^{7}$. En effet, une fois que le régiment a transmis le contrôle qu'il exerçait sur le territoire $(t h \hat{\imath})$ à un nouveau régiment, ses membres entamaient une carrière individuelle au sein des conseils des anciens. Cet accomplissement personnel dépendait des qualités de chacun des individus qui poursuivaient ainsi l'accumulation de richesse et de prestige conduisant au dernier conseil, celui des adultes des quatre béliers ${ }^{8}$. La participation aux conseils ne s'ouvrait, officiellement, qu'aux adultes, parents d'un enfant au moins : athuri ou atumia. Il fallait donc être passé par les différents degrés d'âge qui ponctuaient l'insertion sociale des enfants, des jeunes gens et des jeunes adultes. Cette hiérarchie des conseils était associée à deux positions de prestige : le mûramati et le mûthamaki (Droz 1999).

La désarticulation de la reproduction sociale et l'exacerbation concomitante de l'individualisme s'expliquent par l'impact de la colonisation. Or, si l'administration coloniale a interdit la succession générationnelle par crainte de l'agitation politique qu'elle était censée susciter, ce sont les missionnaires qui ont condamné le régime des classes d'âge en raison des «cérémonies païennes » qui accompagnaient la circoncision ${ }^{9}$. Cette désarticulation a été

7. G. KERSHAW (1997: 65-68). Nous n'avons malheureusement que peu d'informations sur la trajectoire de vie des femmes kikuyus; encore moins au sujet des conseils des femmes qui devaient également jouer un rôle dans la reconnaissance du statut de mûramati (LAMBERT 1956 : 95, 98-99 ; MAAS 1986: 37, 54-56 ; PEATRIK 1994 : 25-26 ; BRINKMAN 1996: 30-81; KERSHAW 1997: 23-26, 117 , 126).

8. Un autre conseil aurait existé, le kiama kia ûkûrû, mais nous disposons de très peu d'informations à ce sujet (HOBLEY 1922 : 150-152 ; LEAKEY 1977 : 8-9 ; KERSHAW 1997: 67 ; PRINS 1953).

9. Notons que le désœuvrement des «guerriers », condamnés à ne « rien » faire a contribué, par la disparition des raids maasaïs et kikuyus, à la disparition du régime des classes d'âge. 
fortement accélérée par la Guerre civile. En effet, l'administration britannique a tenté de mutiler, voire de détruire la reproduction sociale kikuyu au moyen de techniques contre-insurrectionnelles et grâce aux connaissances ethnographiques, ainsi qu'à la verve revivaliste de missionnaires protestants. La disparition du régime générationnel kikuyu, ainsi que celui des classes d'âge (mariika), explique l'affaissement du régime de parenté sur la famille patrimoniale avec comme conséquences l'amenuisement des réseaux de soutien et la diminution de la sécurité de subsistance (Droz \& Sottas, à paraître). Les deux régimes structurants avaient tendance à contrôler les prédispositions fissionnelles que subissait la «société kikuyu» en raison, d'une part de son expansion territoriale et démographique, d'autre part du principe lignager et de l'ethos du mûramati. Un seul de ces régimes nous intéresse ici, celui des classes d'âge créées par le rituel de circoncision.

\section{Le régime des classes d'âge (mariika)}

Avant la période coloniale, une très forte solidarité unissait les membres d'une même promotion, tant masculine que féminine. Cette alliance des pairs contrait les solidarités lignagères et offrait une certaine indépendance aux jeunes adultes dans l'établissement de leurs relations sociales. Cellesci n'étaient plus seulement dictées par le régime de parenté, mais également par la solidarité interne au riika. Néanmoins, les liens de parenté reprenaient progressivement leur position dominante. En effet, après la période de l' «adolescence » qui précédait le mariage, puis celle où le jeune homme acquérait le statut de guerrier, caractérisée par l'importance attachée à la classe d'âge, la trajectoire de vie qui menait à l' « homme accompli » passait par l'insertion individuelle au sein de la hiérarchie des conseils des anciens. Ainsi, « dès l'entrée de quelques-uns de ses membres dans le premier conseil des anciens, le riika ou classe d'âge commençait à perdre sa cohésion, l'accession à ce conseil et aux conseils suivants étant une affaire personnelle et non pas corporative. Elle dépendait, d'une part, des qualités personnelles de chaque homme [...], d'autre part, du paiement d'un droit d'entrée en chèvres, et aussi du hasard, c'est-à-dire, de la naissance et de la circoncision des enfants [...]. Par le jeu compliqué de ces différents facteurs, une classe d'âge d'hommes mûrs pouvait très bien être complètement dissoute, ses membres étant dispersés dans plusieurs "degrés d'ancienneté" » (Buijtenhuijs 1971 : 23-24). Pourtant, cette dissolution n'était jamais totale et la solidarité de riika persistait tout au long de la vie. Elle était particulièrement forte pour les personnes qui avaient été circoncises lors d'une seule et même cérémonie, car ils se considéraient comme des agnats et respectaient entre eux l'interdiction de l'inceste.

Pour comprendre l'importance de la circoncision, tant féminine que masculine, il est nécessaire de saisir le sens de la notion d'être humain chez les Kikuyus : "La reconnaissance comme personne ne s'obtient donc guère 
par le seul fait d'exister, d'être né parmi les enfants des hommes. [...] La naissance selon la chair ne constitue qu'une condition minimale préalable d'accès au statut de mûndo [être humain]. Elle appelle une confirmation sociale sans laquelle l'être humain demeure privé de ce qui en fait une personne. Un enfant, un homme ou une femme non initiés ne comptent littéralement pas. Ils ne sont sujets d'aucun droit, ni sur le plan sexuel et social, ni sur celui de la politique, de l'économie ou de la religion. On s'y réfère tantôt comme à des animaux (nyamo), tantôt comme à des objets inanimés (indo), jamais comme à des personnes (ando) [...]» (Neckebrouck 1983 : 384-385). Nuançons cette affirmation qui pourrait laisser croire que les enfants ne sont rien. C'est tout le contraire, car l'enfant comporte en lui tout le sens de la vie. Sa présence est la condition du succès de l'homme accompli, pour autant que l'enfant grandisse et devienne une personne (Droz 2000).

Et c'est là que l'importance de la circoncision apparaît : elle octroie à l'enfant le statut de personne et l'autorise à perpétuer le phylum familial, présence continue de la famille à travers les générations successives représentée par la récurrence des noms propres. Rappelons que cette entrée dans l'âge adulte représente également une affirmation de l'identité ethnique: être circoncis à la manière des Kikuyus signifie que l'on est un(e) «vrai(e)» Kikuyu. La circoncision confirme également l'accomplissement des parents et leur permet d'atteindre un autre statut social, celui d'ancien. Robert Buijtenhuijs (1971 : 33) souligne le néant social qui environnait l'incirconcis qui « $[\ldots]$ ne pouvait pas posséder de biens, ni avoir sa propre résidence, ni participer aux danses et aux combats, ni porter les cheveux longs, ni manger de la viande, ni boire ou manger avant que les circoncis en sa compagnie n'aient bu ou mangé, ni se baigner en aval de l'endroit où se baignait le circoncis, etc.». Ces mêmes traits ressortent des propos des jeunes hommes et des jeunes femmes que nous avons recueillis. Les noncirconcis ne peuvent faire face aux tâches que l'on attend d'eux. Ils sont incapables de contrôler leur nature (ils réfrènent mal leur énurésie), leur sexualité (elle est débridée pour les filles ou vaine pour les garçons qui s'abandonnent à la masturbation solitaire) et leurs pratiques sociales (irrespect des anciens et de leur conjoint).

\section{La circoncision masculine (irua)}

Aujourd'hui, l'unité de la promotion tend à disparaître en raison des transformations du rituel qui, de commun aux deux sexes, devient individuel ou secret pour répondre à la condamnation de l'État et de certaines Églises. De même que le régime générationnel, le régime des classes d'âge a presque complètement disparu des pratiques sociales, plus particulièrement parmi les jeunes hommes. En effet, au cours de la période coloniale, la circoncision masculine a peu à peu cessé de s'effectuer en groupe sous l'influence dis- 
crète des Missions et au nom de l'hygiène (Buijtenhuijs 1971 : 263 ; Leakey 1952 : 79).

S'il est vrai que la solidarité des promotions s'est presque effacée, au début des années quatre-vingt-dix, certains jeunes hommes, dont nous avons recueilli le témoignage (Droz 1995, 1999), se sont encore fait circoncire en groupe et de façon «traditionnelle » précisent-ils. Ils se sont rendus sur les berges d'une rivière pour s'y baigner, tôt le matin. Vêtu de peaux de bête, le circonciseur est apparu soudain en sautant pour remplir son office. Ensuite, les jeunes hommes se sont séparés pour rentrer chez eux. Ils étaient attendus par l'ensemble de la famille, par des voisins et des amis, réunis pour fêter le joyeux événement. Ce rituel conserve de fortes similitudes avec la circoncision précoloniale telle que Louis Leakey (1977 : 587-686) ou Jomo Kenyatta (1978: 134-154) l'ont décrite. Il indique la persistance de pratiques kikuyus relativement peu influencées par la modernité. Pourtant, ces événements, censés favoriser la cohésion sociale et aller à contrecourant des tendances fissionnelles propres au régime de parenté, n'assurent plus leur fonction unificatrice puisque ces rituels sont confinés à certains groupes restreints. Ils confirment l'éclatement de la «société kikuyu »en une multitude de groupes différents dont certains jouissent encore d'une solidarité issue d'une circoncision commune alors que d'autres, circoncis au dispensaire, ne disposent pas d'un groupe d'entraide fondé en circoncision. Si la "société kikuyu » n'a certes jamais constitué une entité homogène, les groupes locaux partageaient pourtant un ensemble de pratiques sociales (circoncision, transmission générationnelle) et un ethos commun (mûramati) qui favorisaient une certaine cohésion sociale.

Ainsi, la grande majorité des jeunes hommes que nous avons interrogés n'ont pas participé à un tel rituel. Généralement, lors des vacances de Noël, l'incirconcis demande à son père — ou à son oncle — d'organiser sa circoncision. Le père en parle à sa femme pour qu'elle cuisine en conséquence ; il achète des boissons, brasse de la bière de miel et abat un mouton pour l'occasion. La nouvelle se transmet rapidement à l'ensemble de la famille patrimoniale et au voisinage. Chacun apporte son aide et participe à la fête. Le jeune homme choisit un parrain de circoncision (mûtiri) parmi ses pairs et se rend au dispensaire, après avoir reçu l'autorisation de son père ou/et de son oncle maternel. C'est là qu'il se fera exciser le prépuce par un infirmier, seul ou en présence de son parrain. Parfois, plusieurs jeunes hommes sont circoncis successivement - mais pas collectivement — sans que cela n'implique ni droits ni devoirs entre eux, au contraire de ceux qui liaient les membres d'un même riika. Le retour chez soi reste toujours source de joie pour la famille et donne lieu à une fête au cours de laquelle les vieilles femmes dansent le ndumo et les jeunes circoncis organisent des danses. Le nouveau circoncis ne participe que peu aux réjouissances, car, affaibli par l'opération, il se couche dès que possible pour reprendre des forces et affronter la douleur dans l'intimité. En général, une ou plusieurs jeunes filles s'occupent de lui au cours de sa convalescence, pendant que sa mère 
reste à l'écart, suivant en cela les pratiques traditionnelles qui voulaient que les parents n'interviennent pas pendant la période de réclusion des jeunes circoncis.

Une fois rétabli, le jeune circoncis adopte une attitude respectueuse des anciens, il se comporte en « homme responsable» et est considéré comme tel. En outre, la circoncision lui ouvre le monde des relations amoureuses. Alors qu'elles le dédaignaient et l'ignoraient lorsqu'il n'était qu'un kîhî̀ (jeune garçon), les jeunes filles s'empressent autour de lui. Dès le retour du dispensaire où il a subi l'opération, le jeune circoncis devient un amant potentiel. Les amourettes commencent et rappellent le nguîko — pratique amoureuse sans pénétration - qui caractérisait la vie sexuelle des jeunes circoncis(es).

Toutefois, certains jeunes hommes kikuyus ne sont pas circoncis. Ils tentent de garder ce « détail » secret, tout en espérant se marier à une femme compréhensive $^{10}$. Mais si les jeunes voisins l'apprennent, ils procèdent à une circoncision forcée comme en témoignent les divers articles de journaux les relatant. Ainsi sous le titre de «Touts Have Vendor Circumcised by Force » le quotidien Nation ${ }^{11}$ écrit :

«A news vendor in Nanyuki town spent his Christmas in bed on Saturday after touts had him circumcised by force. The 20-year-old man was accused of enjoying the "same female facilities" as circumcised men. A tout, Mr. Bonafus Mathenge said after the touts suspected that the man had not been circumcised, they caught and inspected him and proved their suspicions right. As the touts gathered to escort the man to the hospital, curious on-lookers gathered round the vehicle. The touts contributed Sh. 300 and escorted the victim to the Nanyuki District Hospital singing Kikuyu circumcision songs. After the circumcision at the hospital, the vendor was taken home. His unsold newspapers were handed over to his colleagues to sell. The touts are contributing Sh. 100 daily for the upkeep of their "patient" and plan to buy him bedding. »

Faut-il voir dans cette circoncision forcée les traces d'une solidarité de la «promotion» des vendeurs de journaux envers l'inadvertance coupable de la famille de l'incirconcis ? Ou plutôt le refus de partager les female facilities avec des non-circoncis? Dans les deux cas, l'accès aux relations sexuelles reste bien le critère distinctif entre les circoncis et les non-circoncis, donc la marque de l'âge adulte.

La circoncision masculine implique des changements d'attitudes immédiats, car les comportements asociaux que peut se permettre le jeune garçon (kîhî̀) sont explicitement interdits au jeune circoncis. Par exemple, les jeunes garçons agissent parfois comme des chenapans: ils dérobent des

10. Le comportement prescrit pour une femme qui découvre, lors de la nuit de noces, que son mari n'est pas circoncis est de hurler au scandale et de réclamer le divorce en affirmant se refuser à avoir des relations sexuelles avec un enfant. L'épouse d'un Kikuyu non circoncis peut ainsi légitimement divorcer et elle sera soutenue dans sa démarche par sa famille et l'opinion publique.

11. Daté du 27.12.1993. 
poules ou des cannes à sucre, ils sont irrespectueux envers les adultes et les anciens, ils se moquent impertinemment des passants, etc. Un jeune circoncis ne pourra plus se permettre ces comportements. Il évitera tout contact avec les jeunes garçons incirconcis qui l'accompagnaient jusqu'alors. De même, les enfants non-circoncis, lorsqu'ils ne sont pas à l'école, aident leur mère à puiser de l'eau. Ils utilisent des brouettes de fortune pour transporter les bidons et transforment cette corvée en une course poursuite. Il n'est pas rare d'entendre les cris que suscitent ces joutes et d'observer l'excitation des jeunes garçons. Un circoncis dédaignera ce mode de portage et évitera de se rendre à la rivière ou à la pompe pour ne pas être confondu avec les bandes de jeunes insolents. S'il se trouve dans l'obligation d'aider sa mère, malade ou surchargée, à puiser de l'eau, ou s'il vit seul — sans femme... - sur une parcelle, il transportera noblement le bidon sur une épaule. S'il en a les moyens, il achètera des bidons d'eau au charretier ou sollicitera l'aide d'une jeune fille qui lui évitera cette corvée dégradante.

En dépit de son individualisation, la circoncision masculine reste « le » rite de passage pour les jeunes hommes kikuyus, car elle consacre, aux yeux de tous, l'entrée dans l'âge adulte du jeune homme. Elle est l'occasion pour la famille patrimoniale de reproduire ses relations sociales avec les voisins et une fraction de la famille étendue puisque tout un chacun est invité à la fête. Ainsi, la qualité de l'accueil reproduira la position de la famille patrimoniale dans la communauté et dans la famille étendue.

\section{La circoncision féminine (irua)}

Dans les missions de la Church Missionary Society, la clitoridectomie est restée - à une exception près — tolérée, contrairement aux autres tendances protestantes (Church of Scotland Mission et Africa Inland Mission). Les missionnaires anglicans ont introduit un rituel tronqué qui autorisait l'opération physique mais pas les pratiques qui l'accompagnaient. La description qu'en proposent Robert Strayer (1978: 145) et Jocelyn Murray serait à comparer aux circoncisions «traditionnelles» pour saisir la modification profonde du sens du rituel induite par la médicalisation de la pratique: «The ceremonies were held at homesteads with only the girls and Christian women present. No hymns were sung; there were prayers, but little ceremony. One or two women gave instruction to the girls in the style of exhortation. Such ceremonies must have been rather bleak proceedings and very much more painful to the girls than an operation performed during the course of an emotionally charged ritual. » Bien que ces cérémonies n'aient pas connu une grande vogue dans les années trente, ce sont ces bleak proceedings que rappellent les clitoridectomies des années soixante-dix pratiquées près de Nairobi par des Kikuyus. Dallas Browne (1991) souligne d'ailleurs le fait que ces groupes s'isolent - pour pratiquer la clitoridectomie ou 
même en parler — des autres Kikuyus connus pour l'avoir rejetée. Il s'agit de la même scission que nous avons décelée parmi les jeunes Kikuyus du plateau de Laikipia. Les familles qui pratiquent la clitoridectomie aujourd'hui restent très attachées à cette pratique qui renferme, pour elles, la condition nécessaire pour devenir une «vraie» femme kikuyu, alors que les familles qui ne la pratiquent plus considèrent la circoncision féminine comme une coutume désuète, indigne des Kikuyus «civilisés ». Pour ce dernier groupe, la clitoridectomie appartient à la sphère des peuples primitifs et est considérée comme un frein au développement.

Chez les femmes, la circoncision a subi une évolution plus profonde que chez les hommes. L'interdiction officielle (1982) de la clitoridectomie n'a pas mis fin à sa pratique, elle l'a simplement repoussée dans le secret ou la discrétion. Il est fréquent de lire dans la presse que des chefs de région ont reçu des réprimandes en raison de leur manque d'assiduité à lutter contre la circoncision féminine, ou que des circonciseuses sont parfois condamnées par les tribunaux. Parce qu'elle est interdite et secrète, la clitoridectomie a lieu au sein de la famille étendue et non plus en groupes nombreux comme cela se faisait encore à la fin des années soixante-dix (Browne 1991 : 248250). Deux ou trois jeunes filles sont réunies avec leur marraine de circoncision et elles se rendent à une rivière à l'aube pour procéder à l'opération. Une circonciseuse est invitée pour l'occasion et officie dans le secret. Les nouvelles circoncises reviennent ensuite chez elles sans qu'une fête ne soit organisée publiquement. La nouvelle du succès de l'opération reste à l'intérieur de la famille et parmi les proches voisins. Il n'existe pas de passage à l'âge adulte, reconnu socialement, comparable à ce qui se produit pour les jeunes hommes et l'on peut voir là un élément qui participe à la dégradation du statut de la femme kikuyu. En effet, une suspicion quant au statut adultité de la femme voit le jour, puisque certaines ne sont pas circoncises et restent donc des «enfants ». En revanche, une solidarité de promotion semble se maintenir entre les jeunes filles circoncises ensemble, mais celleci ne fait que renforcer les liens familiaux et ne joue plus le rôle fusionnel des classes d'âge.

En dépit de l'absence d'une reconnaissance affichée de leur nouveau statut, les femmes circoncises sont respectées et entourées d'une aura de maturité. Les hommes et les femmes les considèrent comme prêtes au mariage. On les tient pour être au fait de l'ensemble des tâches qui les attendent en tant que responsables d'une unité domestique. En outre, elles sont censées respecter leur mari en particulier et les anciens en général. Les rumeurs qui courent à leur sujet sont équivoques. Certains jeunes hommes affirment que leur sexualité est plus « chaude » que celle des filles incirconcises. D'autres jeunes hommes prétendent que ce sont les filles non circoncises qui sont les plus libres sexuellement. Mais tous craignent la sexualité incontrôlée des jeunes filles qui n’ont pas été circoncises.

Soulignons que des rumeurs sur la sexualité incontrôlée des incirconcises couraient dans les années trente déjà, puisque les premières prostituées 
kikuyus étaient censées être des jeunes filles incirconcises (ibid. : 258). Nos interlocuteurs nous ont rapporté ${ }^{12}$ que les filles non circoncises deviennent souvent des prostituées dans les villes. Les parents sont tenus pour responsables du triste destin de leur fille : elles sont biologiquement incapables de réfréner leurs désirs sexuels, puisqu'elles ne sont pas circoncises affirme l'imaginaire kikuyu. Certains hommes se refusent à épouser des filles non circoncises prétendant qu'elles ne savent pas tenir un ménage et qu'elles sont frivoles ou incontrôlables. Ils trouveront là un prétexte rêvé pour éviter d'épouser une fille enceinte de leurs ouvres et rejeter la responsabilité de la grossesse sur les parents de la jeune fille: «S'ils l'avaient circoncise, elle ne se serait pas laissée aller. » De plus, ils soulignent les doutes qu'ils ont quant à leur paternité : «Chacun sait qu'une fille non circoncise se donne à n'importe qui. » Il est donc légitime de penser, comme l'affirment de nombreux Kikuyus, que le rejet de la circoncision féminine a contribué à augmenter le nombre de filles-mères.

Selon le climat politique, la circoncision féminine a pu trouver une nouvelle popularité comme le montre l'attachement à cette pratique lors de la Guerre civile ou lors de l'épisode des serments (Davison 1989 : 202 ; Murray 1974 : 343). Jocelyn Murray (1974: 352) estimait que $75 \%$ (> $23 \%$ pour la région de Nairobi) des adolescentes kikuyus étaient circoncises en 1972, alors que Dallas Browne évaluait le nombre de femmes circoncises dans un quartier de Nairobi à $40 \%$ à la fin des années soixante-dix ${ }^{13}$. A la suite de l'interdiction officielle, intervenue en 1982, nous ne disposons plus de données statistiques sur l'étendue du phénomène, mais parmi les migrants kikuyus du plateau de Laikipia (originaires des districts de Nyeri et de Mûrang'a), une estimation grossière laisse penser qu'au milieu des années quatre-vingt-dix, près de la moitié des jeunes filles ne sont pas circoncises.

La circoncision féminine est en perte de vitesse parmi les migrants du plateau de Laikipia où nous avons mené cette recherche. Néanmoins, nombre de nos interlocuteurs (tant des hommes que des femmes) avaient l'intention de faire circoncire leurs futures filles. La perspective d'une disparition progressive de la clitoridectomie ajoute un supplément d'anxiété au sentiment qu'ont les parents de perdre leur influence sur le devenir de leurs filles. Un emploi en ville ou dans une grande propriété foncière dégage la jeune fille du contrôle de ses parents. La perspective de voir naître un enfant sans que le père le reconnaisse inquiète les parents et le mariage devient toujours plus incertain (Droz 1999). Les Kikuyus, dont l'affiliation religieuse n'exige pas l'abandon de la circoncision féminine, ne savent que faire pour pallier les dangers que laisse entrevoir l'avenir de leur fille. Faut-

12. Voir également NecKebrouck (1978: 230-242) et surtout le travail de White (1990) sur la prostitution à Nairobi depuis le début du siècle.

13. Les différences entre ces estimations s'expliquent par les particularismes locaux qui caractérisent les Kikuyus et par l'influence des Églises. 
il respecter la coutume en s'exposant à des amendes et à l'opprobre de la part de l'État et des étrangers ? Ou faut-il afficher sa modernité au risque de voir sa fille se prostituer ou de ne pouvoir la marier?

Irua

L'évolution récente de la circoncision tant féminine que masculine tend à donner raison à Robert Strayer $(1978$ : 144) et à Jocelyn Murray lorsqu'ils remarquent que : "To the Kikuyu, the basic and essential part of the initiation ceremony, the sine qua non of adult status, was the actual physical operation itself, rather than the rituals surrounding it. » Ces propos seront repris par Dallas Browne quelques années plus tard. Cette position contredit les théories ethnologiques des rituels qui considèrent l'opération chirurgicale comme un simple moyen d'inscrire le statut adultite sur — ou dans - le corps, mais non pas comme l'aspect essentiel du rituel, conçu comme un rite de passage. Pour les Kikuyus, outre le signe indubitable de la «kikuyuité », cette opération mettait à l'épreuve la résistance physique du candidat et montrait sa capacité à contrôler son corps et à faire face à la douleur, devant l'ensemble de la communauté. Les conséquences des réactions du candidat lors de l'intervention chirurgicale se répercutaient sur l'ensemble de sa famille étendue. Elle perdait la face si son rejeton montrait quelque signe de peur ou de souffrance. En effet, devenir adulte signifiait savoir supporter la douleur, car, pour les Kikuyus, la souffrance faisait partie intégrante de la vie. Il faut donc voir dans cet apprentissage de la souffrance la raison de l'absence d'anesthésie lors de la circoncision masculine en milieu hospitalier: l'opération doit être douloureuse pour conserver son sens ${ }^{14}$. Rappelons encore que la circoncision n'était pas le seul moment où le corps servait de support à un apprentissage de la souffrance : avant la circoncision, le percement des oreilles (matû) et l'extension des lobes (pour les jeunes filles) était très douloureux rapportent les femmes interrogées par Jean Davison et, après la circoncision, les scarifications « esthétiques » de l'abdomen et du Mont de Vénus ne devaient pas se faire sans douleur ${ }^{15}$.

Depuis la fin des circoncisions en présence de l'ensemble de la communauté, la charge symbolique que comportait le rituel s'est condensée dans l'opération physique. Elle n'est plus aujourd'hui le simple signe extérieur de l'âge adulte, mais elle représente le rituel in extenso, indépendamment

14. «Pain and adversity are normal features of adult life. One major lesson taught to girls undergoing this operation [clitoridectomy] was that pain and hardship must be endured» (BROWNE 1991: 252-253). Certes, certains jeunes hommes demandent parfois une anesthésie, mais ils tentent de garder ce «détail » secret, car ils risquent de devenir la risée de leurs camarades.

15. L. S. B. LEAKEY (1977: 379-381) et J. DAVISON (1989: 64-65, 95-96, 113-114). Sous l'influence des missionnaires, les oreilles de nombreuses femmes ont été recousues, car il s'agissait d'une coutume «païenne» dont tout bon chrétien devait se défaire. 
du fait qu'il ait perdu la plupart de ses aspects sociaux. La comparaison entre la coupure du cordon ombilical et la circoncision du prépuce ou du capuchon clitoridien (Browne 1991 : 253) nous paraît bien condenser le sens que comporte aujourd'hui l'opération physique : il s'agit d'une intervention chirurgicale qui exprime la renaissance dans une nouvelle position sociale, bref d'un rite de passage (Van Gennep 1909). La fête qui accompagne le retour du circoncis et la considération de la famille étendue, tant pour le jeune homme que pour la jeune fille, maintiennent une inscription sociale amoindrie du rituel. Il ne s'agit plus d'un fait social total, mais de l'institution d'un nouvel être social personnel (Bourdieu 1986).

L'école a partiellement remplacé les promotions pour ce qui concerne la vie de groupe : c'est là que s'exerce la pression sociale qui incite le jeune garçon à se faire circoncire. De plus, l'école sépare également les jeunes filles circoncises des écolières «modernes », puisque deux groupes se forment qui reproduisent la division qui existe entre les femmes kikuyus. Dès son arrivée en Form One (neuvième année scolaire), le jeune garçon non circoncis sera soumis à des brimades et souffrira du mépris de ses pairs. Ceux-ci le contraindront à se dévêtir sous les quolibets ou à laver le linge de l'ensemble de la classe. Il subira diverses formes de racket et sera fréquemment battu par les circoncis. Ces mauvais traitements continueront parfois avec l'accord tacite des enseignants, mais le plus souvent à leur insu - jusqu'à ce que l'enfant abandonne l'école ou se fasse circoncire.

Si la circoncision masculine reste le moment de l'ancrage du jeune adulte dans la société, la circoncision féminine a un effet moindre, car celleci ne se pratique plus systématiquement et ne peut plus s'afficher publiquement. Pourtant, ce n'est qu' une fois circoncis que le jeune adulte sera considéré comme responsable potentiel de ses actes. Il pourra alors entamer le parcours qui le mènera à l'accomplissement de soi, en tant que mûramati pour l'homme et en tant que mère de nombreux enfants, gestionnaire efficace de l'unité domestique et belle-mère bienveillante mais sévère pour la femme. En revanche, l'insertion au sein d'un groupe de pairs a complètement disparu pour les hommes, car l'opération s'est individualisée. Alors qu'elle s'est trouvée rejetée dans la clandestinité pour les femmes, une certaine solidarité de la promotion persiste, mais entre des jeunes femmes déjà emparentées. Il ne s'agit donc pas de la constitution d'une classe d'âge représentant un voisinage, mais plutôt de la réactualisation de relations de parenté. En d'autres termes, d'un renforcement du régime fissionnel de la parenté au détriment du régime fusionnel des classes d'âge. Comme le dit une interlocutrice âgée, il n'y a plus de riika chez les Kikuyus : "Ya, the riika was destroyed because there are some people who don't get circumcised $[\mathrm{Hmm}]$. They are following the education, the present education now. »

\section{Les conséquences de l'interdiction de la circoncision féminine}

L'interdiction promulguée par Daniel Arap Moi en 1982 a renforcé les rumeurs qui courraient sur l'appartenance du président à des « sectes » reli- 
gieuses protestantes revivalistes. Il ne s'agissait plus de détruire les Kikuyus pour leur voler la terre comme lors de la période coloniale, mais de confisquer le pouvoir politique qu'ils avaient monopolisé après l'Indépendance. Faut-il voir dans les rumeurs qui courraient au milieu des années quatrevingt-dix sur l'appartenance du président Moi à une secte de suppôts de Satan (Droz 1997a) la perpétuation des rumeurs précoloniales qui liaient vol de la terre et disparition des Kikuyus ? En effet, si l'interdiction de la circoncision féminine, par les missionnaires ou par le président, mettait en danger l'avenir de la population kikuyu en condamnant les enfants à ne jamais devenir adulte, les rumeurs sur les enlèvements d'enfants pour des sacrifices sataniques menacent la survie des familles des migrants du plateau de Laikipia.

La circoncision féminine paraît être en diminution, mais aucun abandon de la pratique n'est prévisible. En effet, tant les jeunes femmes que les jeunes hommes que nous avons interrogés ont des opinions tranchées sur la circoncision féminine qui semblent dépendre de leur affiliation religieuse. Les «abolitionnistes », presbytériens, pentecôtistes et parfois anglicans affirment que leurs filles ne seront jamais circoncises, alors que les « traditionalistes », catholiques, orthodoxes, indépendants et parfois anglicans, sont farouchement attachés à la pratique. Notons que certains jeunes hommes, encore célibataires, laissent à leur épouse potentielle le choix de circoncire ou non leurs futures filles. Cette polarisation en deux camps qui souvent s'ignorent mutuellement confirme les propos de Dallas Browne (1991: 246) : "Families practicing clitoridectomy live in encapsulated social networks and seldom discuss it with families that disapprove of it. » Cette affirmation s'applique également aux familles qui rejettent cette pratique. Circoncire ou non ses filles divise donc les communautés en deux réseaux relativement indépendants. Chacun méprise secrètement l'autre, considérant soit la circoncision féminine comme une pratique qui cantonne ceux qui la suivent parmi les «primitifs", soit les incirconcises comme des enfants immatures indignes de confiance, bref comme des Kikuyus incomplètes. Dès lors, il est difficile de soutenir des relations suivies, car chaque manquement aux pratiques sociales prescrites devient un prétexte pour rejeter l'interlocuteur dans le camp des «primitifs» ou dans celui des «immatures » et se conforter dans son opinion.

La collaboration qui prévalait entre les femmes au sein de groupes de travail informels (ngwatio) ne peut plus se déployer à une grande échelle et reste confinée aux groupes religieux et aux groupes d'entraide (harambee). Il est vraisemblable que l'adhésion à ces associations suivent la ligne de fracture introduite par les tentatives d'abolir la clitoridectomie. En effet, la pratique de la circoncision féminine ne semble pas dépendre du statut économique de la famille, encore moins de son origine géographique, mais bien plutôt de son affiliation religieuse. Les différentes Églises présentes au Kenya se distinguent selon qu'elles interdisent ou non la clitoridectomie, et l'œcuménisme dont font preuve les Kikuyus face aux diverses affiliations 
religieuses est mis à mal par la position des Églises vis-à-vis de cette pratique. Un nouveau principe de division agit donc entre les femmes d'une même communauté, déjà morcelée par le régime de parenté ou les liens de voisinage $\mathrm{e}^{16}$.

Le détour par la circoncision masculine éclaire d'un jour complémentaire nos propos sur la circoncision féminine. En effet, l'irua ne distinguait pas ces deux pratiques et l'évolution différente qu'elles ont subie soulève quelques questions. Nous sommes face à une dissolution du rituel de la circoncision masculine qui, à l'exception de l'inscription du statut adulte et de la «kikuyuité », perd une partie de ses aspects performatifs proprement sociaux. En effet, les pratiques sociales sont limitées au cercle familial et aux voisins proches et ne concernent plus l'ensemble du groupe local. La classe d'âge n'existe plus, ni pour les hommes, ni pour les femmes, ni pour la reproduction sociale... mais l'ablation du prépuce persiste comme une opération chirurgicale indispensable à tout Kikuyu. Les promotions de circoncisions ne jouent plus qu'un rôle diffus qui s'est reporté sur la réussite scolaire (réussir aux examens ou avoir atteint un certain niveau scolaire permet de créer le sentiment d'appartenance à un groupe qui n'est pas sans rappeler la solidarité qui unissait les riika).

La circoncision masculine conserve donc un sens restreint en transformant l'adolescent en homme responsable. Il n'existe aucun doute quant au statut de l'homme circoncis, contrairement à la suspicion qui enveloppe le statut de la femme - même mère - et surtout ses capacités à contrôler ses «pulsions », ainsi qu'à faire face aux devoirs conjugaux et matrimoniaux. En dépit de la médicalisation et de l'individualisation de la pratique de la circoncision masculine, certains aspects performatifs du rite de passage persistent donc aujourd'hui. On peut se demander si cette médicalisation n'a pas permis son insertion dans une modernité en éliminant les éléments les plus «barbares » - aux yeux des missionnaires en particulier et des Occidentaux en général - tout en conservant une partie de l'efficacité sociale du rituel. L'homme adulte reste complètement socialisé en tant que Kikuyu par l'opération chirurgicale, alors que l'incertitude règne quant à l'appartenance de la femme à la culture : par son inconstance et son défaut d'inscription sociale, l'incirconcise est toujours soupçonnée de participer de l'état de nature. Ainsi, outre l'insidieuse division sociale introduite entre les femmes par les tentatives d'abolition de la circoncision féminine, la disparition partielle de cette pratique a insinué une suspicion généralisée quant à la qualité de la femme kikuyu. Est-elle vraiment une adulte ?

\section{Perspective anthropologique versus individualisme éthique}

Avant de porter un jugement sur les pratiques sociales, il est essentiel de chercher à les comprendre pour espérer pouvoir agir ensuite. Pour ce faire,

16. Soulignons que les tentatives abolitionnistes ne sont qu'un des nombreux éléments (colonisation, Guerre civile, propriété de la terre, travail salarié, etc.) qui ont désarticulé la reproduction sociale kikuyu (Droz 1999). 
quelques remarques nous semblent appropriées en conclusion. Si d'un point de vue humaniste et individuel, il peut sembler éthiquement juste de condamner la pratique de la circoncision féminine - même si elle est acceptée par l'ensemble des participant(e)s au rituel - en se référant aux droits de l'Homme, la question revêt une autre dimension lorsqu'on l'envisage d'un point de vue anthropologique. Il n'est alors plus possible d'évaluer ses seules conséquences individuelles, imaginées à partir de la notion abstraite d'un individu autonome, associée à celle de citoyen d'un État. L'idée de citoyen est au cœur de la construction du concept d'individu comme sujet des droits de l'Homme. Cette idée plonge ses racines dans la naissance de la citoyenneté et de la société civile en Europe au siècle des Lumières et s'ancre progressivement dans les pratiques sociales. L'usage de la citoyenneté nous paraît inséparable de l'existence d'un État de droit accompagné d'assurances sociales qui garantissent au citoyen une certaine sécurité face à l'avenir et lui permettent de se libérer des réseaux clientélistes, bref, de se «désenchâsser» (Polanyi 1983) des contraintes sociales. En effet, sans la sécurité que représentent les assurances sociales, l'individu ne peut acquérir une autonomie décisionnelle, car il reste dépendant de réseaux clientélistes pour pallier les situations de crise (Kohli 1994 ; Mayer \& Müller 1994). Or, en Afrique subsaharienne, ni la notion d'État de droit, ni celle de citoyen n'ont été incorporées dans les pratiques sociales afférentes à ce que l'on nomme aujourd'hui la «gouvernance » (Bayart 1989 ; Neubert 1996). Les deux conditions préalables, nécessaires à l'exercice des droits de l'Homme, sont donc absentes.

Ainsi, au Kenya, l'individu n'existe pas en tant que sujet abstrait des droits de l'Homme, mais plutôt en tant que membre d'une famille, d'un groupe, d'une société singulière. Chaque individu est inséré au sein de divers réseaux familiaux et/ou clientélistes qui dictent des droits et des devoirs inégaux, dépendants de la position particulière et momentanée de chacun. L'égalité et l'autonomie, que la citoyenneté suppose, ne constituent aucunement les caractéristiques de ces réseaux sociaux. Au contraire, l'inégalité leur est constitutive, puisque l'accès différencié aux diverses ressources sociales, économiques ou géographiques est la raison de leur existence. En revanche, une forme d'équité dans les échanges (matériels et symboliques) permet leur reproduction : les échanges de biens et de services qui expriment ces réseaux ne pourraient perdurer si chacun n'y trouvait un avantage. C'est cette insertion sociale — cet « enchâssement » — qui permet de faire face aux situations de crise en l'absence d'un État garantissant un minimum de sécurité physique et sociale à ses citoyens. Mais c'est également cette insertion sociale qui interdit la naissance d'une société civile composée de citoyens libres et égaux, les seuls auxquels peuvent s'appliquer les droits de l'Homme.

Les circoncisions féminines et masculines ont joué un rôle essentiel dans la reproduction sociale kikuyu. La séparation d'un rituel unique en deux pratiques relativement indépendantes, l'une s'individualisant tout en se 
médicalisant et l'autre se dissimulant tout en scindant le groupe social, a conduit au surgissement d'un nouveau principe de division. L'actuelle cohabitation de deux systèmes symboliques opposés, l'un prônant la réalisation de soi par l'adhésion à un ethos inégalitaire mais équitable, l'autre affirmant l'égalité de tous les individus autonomes face à des droits abstraits, conduit à une situation paradoxale. La femme kikuyu en particulier, et sa famille en général, sont confrontées à la présence de deux systèmes de valeurs concurrents et incompatibles: soit la femme conserve une reconnaissance sociale en tant que «vraie » Kikuyu, au prix du secret, de l'illégalité et du stigmate de l'atavisme ou de l'aliénation ; soit elle se «modernise » en devenant la proie de rumeurs peu flatteuses quant à ses capacités de faire face aux tâches conjugales et familiales et en perdant le statut de femme accomplie, voire de Kikuyu. Aucun des termes du dilemme n'offre un statut valorisé à la femme, et cette évolution a fortement altéré les droits dont la femme kikuyu jouissait lors de la période précoloniale, en tant qu'épouse, en tant que membre d'un réseau lignager, d'une classe d'âge, etc. L'interdiction par l'État de la circoncision féminine, au même titre que la modification du droit foncier, que la transformation des pratiques matrimoniales ou que l'introduction du travail salarié ${ }^{17}$, a donc aggravé le statut de la femme en pays kikuyu. En effet, outre la réduction de l'étendue des groupes d'entraide (ngwatio), cette interdiction a contribué à faire disparaître la solidarité qui unissait les membres d'une même classe d'âge et a souvent scindé des lignages ou des communautés locales en séparant les femmes ou les familles selon leur participation aux rituels de la circoncision féminine.

L'interdiction d'une pratique sociale, la clitoridectomie, au nom de l'individualisme éthique contenu dans les droits de l'Homme, n'a pas été accompagnée de son corollaire indispensable: la constitution d'un État social qui aurait pu offrir une alternative aux réseaux sociaux dans lesquels l'individu se trouve «enchâssé » et lui offrir la possibilité de choisir de manière indépendante. Cette «ingérence humanitaire », qui s'apparente à une double contrainte, contribue à désarticuler la reproduction sociale kikuyu, donc à diminuer les recours que les différents réseaux sociaux proposent aux individus (Droz \& Sottas 1997). Elle laisse les Kikuyus, tant homme que femme, en proie aux effets néfastes d'une mondialisation économique, sans qu'ils ne bénéficient ni des aspects positifs d'un État de droit (citoyenneté, assurances sociales, droits de l'Homme), ni de la sécurité que leur offraient les réseaux de solidarité autochtones.

17. Voir F. Mackenzie (1989); Y. Droz (1999); G. Kershaw (1997 : 123-127). Notons que certains auteurs affirment que ces transformations se sont accompagnées d'un gain d'autonomie pour la femme qui peut gérer seule l'argent qu'elle gagne ou le lopin de terre qu'elle possède en son nom propre (BRINKMAN 1996: 45-47). Toutefois, il peut sembler surprenant que la dissolution de l'union matrimoniale soit perçue comme un signe d'amélioration du statut de la femme (DROZ 1997b). 
Dans le cas des Kikuyus, interdire la clitoridectomie signifie intervenir contre la volonté de l'enfant, contre la volonté de ses parents et contre les normes morales, ou l'ethos kikuyu. Une question se pose alors : est-il éthiquement défendable de prôner le respect des droits de l'Homme en général et l'abolition de la circoncision féminine en particulier, sans avoir, au préalable, établit les conditions indispensables à leur exercice ? Certes, cette interrogation est dérangeante, car les conditions préalables à l'exercice des droits de l'Homme comportent la constitution d'un État de droit et la mise en place d'assurances sociales. Deux exigences difficiles à réaliser en Afrique subsaharienne, au vu des moyens financiers et éthiques qu'elles présupposent. Or, si ces conditions d'applicabilité ne sont pas réunies, la revendication des droits de l'Homme revient à extraire l'individu de réseaux qui lui assurent encore une certaine «sécurité sociale» sans lui en garantir une autre, donc à déstructurer la reproduction sociale. Si notre analyse est correcte, ce serait exiger le respect de droits idéaux sans en offrir les moyens de le faire.

Institut universitaire d'études du développement (IUED), Genève.

\section{BIBLIOGRAPHIE}

AHLBERG, B. M.

1991 Women, Sexuality and the Changing Social Order: the Impact of Government Policies on Reproductive Behaviour in Kenya, Philadelphia, Reading; Paris, Montreux; Tokyo, Gordon \& Breach.

BAYART, J.-F.

1989 L'État en Afrique, la politique du ventre, Paris, Fayard.

Bourdieu, P.

1986 «Les rites d'institution », in P. Centlivres \& J. Hainard, eds, Les rites de passage aujourd'hui, Lausanne, L'Age d'Homme : 206-215.

BRINKMAN, I.

1996 Kikuyu Gender Norms and Narratives, Leiden, CNws Publications/Leiden University.

BRowne, D.

1991 «Christian Missionaries, Western Feminists, and the Kikuyu Clitoridectomy Controversy », in B. Williams, The Politics of Culture, Washington-London, Smithsonian Institution Press : 243-272.

Bugeau, F.

1911 «La circoncision au Kikuyu (British East Africa)», Anthropos, 6 : 616-627. 
BuiJTENHUis, R.

1971 Le Mouvement «Mau-Mau»: une révolte paysanne et anti-coloniale en Afrique noire, La Haye-Paris, Mouton.

BuNCHE, R. J.

1941 «The Irua Ceremony Among the Kikuyu of Kiambu District, Kenya », Journal of Negro History, 26 : 46-65.

DAVISON, J.

1989 Voices from Mutira : Lives of Rural Gikuyu Women, Boulder, Lynne Rienner.

DROZ, Y.

1995 Anthropological Outline of a Kikuyu Community (Laikipia District, Kenya), Laikipia Research Programme, Nairobi, University of Nairobi ; Berne, University of Berne.

1997a «Si Dieu veut... ou suppôts de Satan ? Incertitudes, millénarisme et sorcellerie chez les migrants kikuyu », Cahiers d'Études africaines, XXXVII (1), $145: 85-117$.

1997b Compte rendu de l'ouvrage de Inge Brinkman, Kikuyu Gender Norms and Narratives, Cahiers d'Études africaines, XXXVII (3), 147: 717-721.

1998 «Genèse de 1'"ethnie": le cas des Kikuyus du Kenya central », Canadian Journal of African Studies/Revue canadienne des études africaines, XXXII (3) : 253-275.

1999 Migrations kikuyus : des pratiques sociales à l'imaginaire; ethos, réalisation de soi et millénarisme, Neuchâtel, Institut d'ethnologie; Paris, Éditions de la Maison des sciences de l'Homme («Recherches et Travaux»14).

2000 «L'ethos du mûramati kikuyu ; shème migratoire, différenciation sociale et individualisation au Kenya », Anthropos, 95 : 87-98.

Droz, Y. \& SotTAS, B.

1997 «Partir ou rester? Partir et rester; migrations des Kikuyus au Kenya », L'Homme, 142 : 69-88.

A paraître «From Lineage to Confined Family : Transformations of Kinship Relations among Kikuyu Migrants »(manuscrit).

GATHERU, M. R.

1964 Child of Two World; A Kikuyu's Story, New York-Washington, Praeger Publishers.

GunNING, I. R.

1992 «Arrogant Perception, World-Travelling and Multicultural Feminism: The Case of Female Genital Surgeries », Columbia Human Rights Law Review, 23 (2) : 189-248.

Hobley, C. W.

1922 Bantu Beliefs and Magic, London, H. F. \& G. Witherby.

Kanogo, T.

1987 Squatters and the Roots of Mau Mau 1905-63, Nairobi, Heinemann Kenya ; London, James Currey; Athens, Ohio University Press. 
1993 «Mission Impact on Women in Colonial Kenya », in F. BowIE, D. KIRKwood \& S. ARdener, eds, Women and Mission : Past and Present. Anthropological and Historical Perceptions, Providence-Oxford, Berg : 165-186.

Kenyatta, J.

1978 [1938] Facing Mount Kenya: The Traditional Life of the Kikuyu, London, Heinemann.

Kershaw, G.

1997 Mau Mau from Below, London, James Currey ; Nairobi, East African Educational Publishers; Athens, Ohio University Press.

KoHLI, M.

1994 [1989] «Institutionalisierung und Individualistierung der Erwerbsbiographie », in U. BECK \& E. BECK-GERnSHEIM, eds, Riskante Freiheiten, Frankfurt am Main, Suhrkamp : 219-244.

LAMBERT, H. E.

1956 Kikuyu Social and Political Institutions, Nairobi-Oxford-New York, Oxford University Press.

LEAKEY, L. S. B.

1931 "The Kikuyu Problem of the Initiation of Girls », Journal of the Royal Anthropological Institute, 61 : 277-285.

1952 Mau Mau and the Kikuyu, London, Methuen.

1977 The Southern Kikuyu Before 1903, London-New York-San Francisco, Academic Press, 3 vols.

LONSDALE, J.

1992 «The Moral Economy of Mau Mau: Wealth, Poverty and Civic Virtue in Kikuyu Political Thought », in B. Berman \& J. Lonsdale, eds, Unhappy Valley. Conflict in Kenya and Africa. t. II, Violence and Ethnicity, Nairobi, Heinemann Kenya ; London, James Currey ; Athens, Ohio University Press : 315-504.

1995 «The Prayers of Waiyaki; Political Uses of the Kikuyu Past», in D. Anderson \& D. H. Johnson, eds, Revealing Prophets ; Prophecy in Eastern African History, London, James Currey ; Nairobi, East African Educational Publishers; Kampala, Fountain Publisher; Athens, Ohio University Press : 240-291.

1996a " "Listen While I Read": The Orality of Christian Literacy in the Young Kenyatta's Making of the Kikuyu », in L. DE LA Gorgendière, K. KING \& S. Vaughan, eds, Ethnicity in Africa; Roots, Meanings and Implications, Edinburgh, Centre of African Studies: 17-53.

1996b «Ethnicité morale et tribalisme politique », Politique africaine, 61 : 98-115.

MAAS, M.

1986 It is Always a Good Thing to Have Land, Leiden, African Studies Centre (Research Report $n^{\circ} 2$ ). 
MACKENZIE, F.

1989 «Land and Territory: the Interface Between Two Systems of Land Tenure, Murang'a District, Kenya », Africa, 59 (1) : 91-109.

1990 «Gender and Land Rights in Murang'a District, Kenya », The Journal of Peasant Studies, 17 (4) : 609-643.

Mayer, K. U. \& MÜller, W.

1994 [1987] «Individualisierung und Standardisierung im Strukturwandel der Moderne Lebensverläufe im Wohlfahrtsstaat», in U. BECK \& E. BECKGERnSHEIM, eds, Riskante Freiheiten, Frankfurt am Main, Suhrkamp : 265295.

MuORIA, H.

1994 The Gikuyu and the White Fury, Nairobi, East African Educational Publisher.

MurRAY, J.

1974 The Kikuyu Female Circumcision Controversy. With Special Reference to the Church Missionary Society's Sphere of Influence (Ph.D. dissertation), Los Angeles, University of California.

NATSOULAS, T.

1988 "The Rise and Fall of the Kikuyu Karing' a Education Association of Kenya, 1929-1952 », Journal of Asian and African Studies, XXIII (3-4) : 219-233.

NeCKeBROUCK, V.

1978 Le onzième commandement. Étiologie d'une église indépendante au pied du mont Kenya, t. XVII, Immensee, Nouvelle revue de science missionnaire.

1983 Le peuple affligé: les déterminants de la fissiparité dans un mouvement religieux au Kenya central, t. XXXII, Immensee, Nouvelle revue de science missionnaire.

NEUBERT, D.

1996 «Westliche politische Ideen und deren Verwirklichung in Afrika. Demokratisierung und Menschenrechte in Kenia und Ruanda », Peripherie, 61 : 43-64.

PeAtrik, A.-M.

1994 «Un système composite : l'organisation d'âge et de génération des Kikuyu pré-coloniaux », Journal des Africanistes, 64 (1) : 3-36.

1995 «La règle et le nombre : les systèmes d'âge et de génération d'Afrique orientale », L'Homme, $134: 13-49$.

POLANYI, K.

1983 [1944] La grande transformation: aux origines politiques et économiques de notre temps, Paris, Gallimard.

Prins, A. H. J.

1953 East African Age-Class Systems ; Galla - Kipsigis - Kikuyu, GroningenDjakarta, J. B. Wolters. 
Routledge, W. S. \& Routledge, K.

1910 With a Prehistoric People: the Akikuyu of British East Africa, London, Edward Arnold.

SANDGREN, D. P.

1989 Christianity and the Kikuyu; Religious Divisions and Social Conflict, BernNew York-Frankfurt am Main-Paris, Peter Lang.

SinDZINGRE, N.

1977 «Le plus et le moins : à propos de l'excision », Cahiers d'Études africaines, XVII (1), $65: 65-75$.

1979 «Un excès par défaut; excision et représentation de la féminité », L'Homme, XIX (3-4) : 171-187.

STRAYER, R. W.

1978 The Making of Mission Communities in East Africa : Anglicans and Africans in Colonial Kenya, 1875-1935, London, Heinemann.

TIGNOR, R.

1976 The Colonial Transformations of Kenya: the Kamba, Kikuyu and Maasai from 1900 to 1939, Princeton, Princeton University Press.

VAn GenneP, A.

1909 Les rites de passage, Paris, Picard.

WhiTE, L.

1990 The Comforts of Home : Prostitution in Colonial Nairobi, Chicago-London, University of Chicago Press.

RÉSUMÉ

II s'agit ici de présenter les aspects anthropologiques du rituel de la circoncision chez les Kikuyus du Kenya et de nous interroger sur les effets anthropologiques de l'interdiction de la circoncision féminine au Kenya en comparant sa pratique actuelle avec celle de la circoncision masculine. Pour cela, l'ethnographie des classes d'âge kikuyus s'avère être un préalable indispensable. En effet, comprendre une pratique sociale particulière - la circoncision féminine et masculine - exige de connaître les relations qu'elle entretient avec l'ensemble de la reproduction sociale. Q uelques remarques sur le changement de perspective qu'implique cette analyse éclaireront les présupposés individualistes et la conception du citoyen à l'origine des tentatives d'interdiction de la circoncision féminine au nom des droits de l'Homme.

\section{ABSTRACT}

Male and Female Circumcision among the Kikuyu: A Rite of Institution, Social Division and Human Rights. - The anthropological aspects of circumcision ceremonies among the Kikuyu in Kenya are presented; and questions, raised about the anthropo- 
logical effects of forbidding female circumcision, as currently practiced. To compare female with male circumcision, it is indispensable to study Kikuyu age-sets. To understand such a social practice requires knowledge of its relations with social reproduction as a whole. A few remarks about how such an analysis would change perspectives sheds light on individualistic presuppositions and on the conception of citizenship underlying the attempts to outlaw female circumcision in the name of human rights.

Mots-clés/Keywords: Kenya, Kikuyu, circoncision, classe d'âge, clitoridectomie, droits de l'H omme, excision, organisation sociale/Kenya, Kikuyu, circumcision, agesets, human rights, social organization. 